\title{
RKKY Interaction in Coupled Quantum Dots
}

\author{
Z. Bak, R. Jaroszewicz and W. Gruhn \\ Institute of Physics, Jan Długosz University \\ al. Armii Krajowej 13/15, 42-200 Czȩstochowa, Poland
}

\begin{abstract}
Assuming that quantum dots are treated as artificial impurities we consider the Ruderman-Kittel-KasuyaYosida interaction between their localized magnetic moments. We prove that due to the quantum confinement the carriers that mediate interactions can exhibit fractional spectral dimension. Basing on this result we discuss magnetic interactions in coupled system of quantum dots and leads.
\end{abstract}

PACS numbers: $47.53 .+\mathrm{n}, 75.30 . \mathrm{Ds}, 75.75 .+\mathrm{a}$

\section{Introduction}

In artificial nanostructures the control of localized, single spin is of great technological importance for nanospintronic applications. In that respect quantum dots (QD), as the building blocks of nanodevices, seem to be most perspective. Quantum dots are electronic systems which confine a well-defined number of electrons. The total spin of a dot can be integer or half-integer (the latter stands for odd number of confined electrons). Typical nanodevices are composed of a few QD interconnected by leads. Electrons (from a lead or metallic substrate) scattered off QD spins induce an effective spin exchange interaction, the Ruderman-Kittel-Kasuya-Yosida (RKKY) interaction [1]. Magnetic correlations of QDs spins arising from the RKKY interaction are very important as they affect conductivity, an effect of primary importance for spintronics. Magnetic moments of QDs are Kondo (partially) screened, so the strength of the RKKY coupling depends both on the QDs separation and the Kondo screening. The Kondo screening can be tuned by the gate voltage; this effect offers an opportunity to control the strength of the effective RKKY exchange. Such a tuned RKKY interaction between QD moments is deemed as a possible way to couple QD based qubits in construction of universal quantum logic gates. We focus our attention on the study of pure RKKY coupling between two dots joined by a lead, leaving the Kondo contribution aside. Two distinct configurations of interacting QDs are considered; the interaction via electrons of a substrate and the other one via electrons of metallic lead which joins adjacent dots. The problem of formation of long-range magnetic order within a quasi-1D ferromagnetic lead is discussed independently.

\section{Model}

The effective RKKY interaction between the spins of the adjacent QDs is given by $H=J(x) \boldsymbol{S}_{1} \circ \boldsymbol{S}_{2}$. To estimate the effect of RKKY coupling on the magnetic ordering, we should find the spatial dependence of the exchange integral $J(x)$. The latter is determined by the spin polarization around magnetic impurity that arises from low-energy excitation of the mobile charge carriers. As the result of that, the RKKY exchange integrals are determined by the energy spectra of mobile charge carriers. This ascertainment is important as the basic difference between the RKKY interaction in bulk solids and in QDs is that in the latter RKKY is mediated by the confined electrons. However, the effect of confinement is reflected in the energy spectrum of mobile electrons, thus provided that the electron spectrum is known, we should not bother about confinement anymore. For the method we are applying below, it is important that there exists an intimate relation between the electron energy spectrum and the effective density of electrons states (expressed as a function of energy) $n(\epsilon)$. We should stress here that we need the energy spectrum of outer electrons (those within a lead or a substrate), not the spectrum of the QD states [2]. In many real systems the density of states is represented by a simple power law formula [3]:

$$
n(\epsilon) \mathrm{d} \epsilon \approx\left(\epsilon-\epsilon_{0}\right)^{\alpha / 2-1} \mathrm{~d} \epsilon .
$$

The scaling parameter $\alpha$ represents the effective spectral dimension, and as a rule, is different from the geometrical dimension $\mathrm{D}$ of the system. The most important fact is that it can take any real (also fractional or negative) values. For our approach it is enough if $n(\epsilon)$ fulfills the relation (1) in a small energy window close to the Fermi energy. In this approach a single parameter - spectral dimension $\alpha$ - contains all the information about anisotropy of the electron mobility associated with the restrained motion of electrons.

Therefore, it is evident that the indirect magnetic interactions due to the confined electrons are governed by the values of the effective spectral dimension as shown in [4]. The analytical expression for the RKKY-like exchange integral in the case of an arbitrary spectral di- 
mension $\alpha$ is given by the following expression [4]:

$$
\begin{aligned}
& J(r)=J_{0} r^{2-\alpha}\left[J_{\alpha / 2-1}(k r) Y_{\alpha / 2-1}(k r)\right. \\
& \left.\quad+J_{\alpha / 2}(k r) Y_{\alpha / 2}(k r)\right],
\end{aligned}
$$

where $J_{\nu}(x)$ and $Y_{\nu}(x)$ are the Bessel and the Neumann functions, respectively. Equation (2) reproduces the well known formulae for 1D, 2D and 3D systems, however, it is valid for systems in which spectral dimension is a fraction [3]. The variance of $J(r)$ (as a function of interspin distance) is depicted in Fig. 1. The characteristic feature of exchange integrals given by Eq. (2) is their oscillatory behavior and the fact that the leading term of the envelope function of $J(r)$ falls off asymptotically with the interspin distance as $J(r) \propto r^{-\alpha}[4]$. This means that the value of spectral dimension determines the decay of the RKKY exchange and by extracting this dependence from experimental or numerical results we can estimate the effective value of spectral dimension.

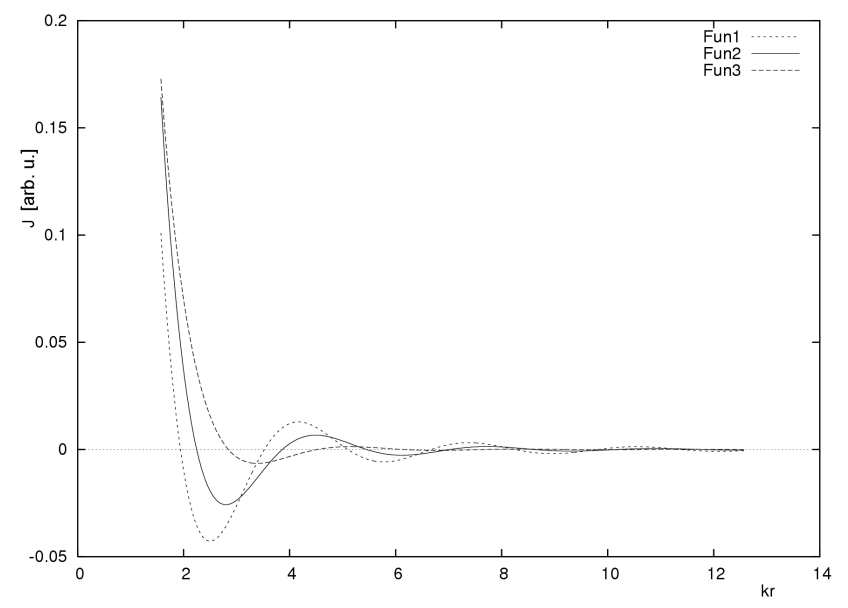

Fig. 1. The RKKY exchange integrals (arbitrary units) for different values of spectral dimension: fun1 $\rightarrow$ $\alpha=2.5$, fun $2 \rightarrow \alpha=3.0$, fun $3 \rightarrow \alpha=4.0$.

To show the implications of this result we should consider the RKKY interaction in a real nanospintronic device. First of all let us consider the electrons confined within a quasi-1D conducting lead joining adjacent QDs. Leads itself are not passive elements. Their electrons transmit the magnetic interaction and in the case of ferromagnetic leads they can spin-polarize the electric current. Thus, thorough consideration of the RKKY coupling within a lead is necessary. In chain geometry dots are connected to each other by quasi-1D wires which set the distance between them. Let us calculate the spectral dimension of a lead in which the confinement potential of electrons is a parabolic function of the distance from a center of the wire. We assume that the " $z$ " and " $y$ " denote the perpendicular directions of the conducting lead. In view of that the Hamiltonian, which describes the electronic structure within the envelope function formalism and effective mass approximation, reads [5]:

$$
H=-\hbar^{2} \nabla^{2} /\left(2 m^{*}\right)+D\left(y^{2}+z^{2} / 2\right)+E_{0} .
$$

Here, $E_{0}$ and $m^{*}$ are the band gap energy and the effective mass at the center of the wire, respectively. $D$ is the curvature of the parabolic potential profile, which is assumed to be infinitely high. In the following we will focus our attention on the single band model with the energy spectrum given by

$$
\epsilon_{n_{x}, n_{y}}^{k}=\hbar^{2} k^{2} /\left(2 m^{*}\right)+\hbar \omega\left(n_{y}+n_{z}+1\right)-\mu,
$$

with $k$ being a $1 \mathrm{D}$ wave vector. The second term in the electron spectrum (4) has the form characteristic of a $2 \mathrm{D}$ harmonic oscillator. In the case of a $1 \mathrm{D}$ harmonic oscillator the density of states $n_{1 \mathrm{D}}(\epsilon)$ is proportional to the energy, i.e., $n_{1 \mathrm{D}}(\epsilon) \propto\left(\epsilon-\epsilon_{0}\right)$ which fitted to the formula (1) gives us $\alpha=2$ [5]. The energy spectrum of electrons confined within a wire consists of two contributions, one of a 2D harmonic oscillator and 1D free electron gas. Thus the effective spectral dimension of a wire with the parabolic confinement potential can be fitted to Eq. (1) by setting $\alpha=5$. This means that electron gas of quasi-1D wire cannot be assumed as a quasi-1D, the preassumption often applied in theoretical descriptions [6], but rather as a 5-dimensional. If spins of adjacent QDs are coupled due to the RKKY interaction mediated via electrons within such a wire the exchange integral is expected to be given by the formula (2) with $\alpha=5$. This result shows that theories based on the assumption that the wire couples QDs spin via 1D RKKY [7] is doubtful.

In all studies of QDs grown over a metallic substrate are assumed to be coupled via $2 \mathrm{D}$ substrate's electron gas. However, in real systems the situation is more complicated, as not only surface carriers contribute to coupling. The population of subsurface electrons always contributes to the RKKY coupling. In this situation the QDs are better described (spectrally) by a $(2+\epsilon)$ D electron gas [8]. The same refers to the RKKY interaction when the vertical stack configuration of QDs is considered. This means that also in this case the assumption of 2D RKKY [6] cannot be accepted.

All the above considerations look as if they were only of speculative nature, to show that it is not so let us point out a system for which there are explicit numerical estimations of the RKKY exchange. The molecular chain that can conduct electrons can serve as the specific form of interdot connection (nanowire). In this respect carbon chains are the most promising, since carbon has a strong tendency to form linear atomic chains, whereas other elements have tendency to create a zigzag structures [9]. Numerical study of the indirect magnetic interaction in the case of the $\mathrm{TMC}_{n} \mathrm{TM}$ (e.g. $\left.\mathrm{CoC}_{7} \mathrm{Co}\right)$ atomic chains, with TM being a transition metal ion, was performed by Durgun et al. [9]. Numerical results predict both ferroor antiferromagnetic configurations of the TM ion spins, arising from the RKKY coupling along the carbon chain. The most interesting result, with respect to the above considerations, is that the decay rate of the RKKY exchange as the function of interspin separation $r$ as extracted from numerical data can be fitted to the simple power law $r^{-\alpha}$ dependence [9]. Value of $\alpha$ turns out to 
be 0.72 for ferromagnetic and 1.43 for antiferromagnetic configurations [9]. This result points out that the spectral dimension is not a constant of structure or of a system, and by changing some external parameters, e.g. electric field or tensile strain we can manipulate the system from outside. Moreover, from the above, it is evident that contrary to the conclusion of paper [9], the RKKY model in its fractional version is applicable in a description of QD systems.

Another aspect of magnetic interactions is the long range magnetic ordering in a wire. Although leads seem to be quasi-1D they can be ferromagnetic. Because in bulk systems long range order cannot arise when the dimension is lower than 2 we should answer why quasi-1D wire can display ferromagnetic ordering. For this reason, let us consider how the dimensionality enters the thermodynamical quantities. For an ideal Fermi/Bose gas the grand potential reads [10]:

$$
\ln \Xi=\int_{0}^{\infty} n(\epsilon) \ln \left(1 \pm \mathrm{e}^{-\beta \epsilon}\right) \mathrm{d} \epsilon .
$$

From Eq. (5) one can easily deduce that all the information about the dimensionality of the actual system enters thermodynamical formulae via the density of states $n(\epsilon)$. Thus, as we can see from the definition of $n(\epsilon)$ the thermodynamical evolution of any system depends on its spectral dimension. As the effective spectral dimension of quasi-1D wire can exceed 3 it is evident that the long range ordering is possible.

\section{Summary and discussion}

There is a widespread conjecture that the dimension of position space (geometrical dimension) and the dimension of dynamical space (spectral dimension) should be both equal and integer. However, there is an experimental evidence that in low-dimensional systems the vibrational or electron density of states as extracted from measurements correlate with those predicted by Eq. (1) with fractional $\alpha$. We have proved that both the RKKY and thermodynamical phenomena are governed by the spectral dimensionality. Quantum dots and leads joining them are not exceptions. Fractional dimensional- ity should be present in some of those systems and up to date approaches to RKKY coupling are only over-simplifications of real systems. We should mention here that the results obtained above are valid provided that interdot tunneling is rather weak. If the tunneling is strong the electron states on either QD become hybridized and form a molecular state [11]. As to the practical implications of our study let us note that although the main results were derived for the special one it seems plausible that predicted effects can realize in any case of coupled QD. Further we believe that our study through the derivation of analytical results can be of considerable help for experimentalists when rapid and accurate estimates of magnetic exchange integrals are needed. Finally, we hope that our results can be helpful in tailoring properties of spintronic devices. In conclusion, we believe that our results not only point the way to a new understanding of magnetism of QD systems but also give practical indications for engineering of fractally structured electronic devices. Even if the considered model does not fit exactly most QD systems it is still useful as it gives a deeper insight into the mechanisms of magnetic interaction in these systems.

\section{References}

[1] C.H. Chung, G. Zarand, P. Wolfle, Phys. Rev. B 77, 035120 (2008).

[2] B. Szafran, E. Barczyk, F. Peeters, S. Bednarek, Phys. Rev. B 77, 115441 (2008).

[3] Z. Bak, Phys. Rev. B 68, 064511 (2003).

[4] Z. Bak, R. Jaroszewicz, W. Gruhn, J. Magn. Magn. Mater. 213, 340 (2000).

[5] Z. Bak, Solid State Commun. 118, 43 (2001).

[6] M. Yang, S.S. Li, Phys. Rev. B 74, 073402 (2006).

[7] P. Durganandini, Phys. Rev. B 74, 155309 (2006).

[8] Z. Bak, Czech. J. Phys. 53, 5 (2003).

[9] E. Durgun, T. Senger, H. Sevincli, H. Mehrez, S. Ciraci, Phys. Rev. B 74, 255413 (2006).

[10] Wu-Sheng Dai, Mi Xie, Phys. Lett. A 311, 340 (2003).

[11] Y.S. Liu, X.F. Yang, X. Zhang, Y.J. Xia, Phys. Lett. A 372, 3318 (2008). 